\title{
CRISPR/Cas9-mediated targeted mutagenesis of GmSPL9 genes alters plant architecture in soybean
}

\author{
Aili Bao1, Haifeng Chen', Limiao Chen', Shuilian Chen', Qingnan Hao', Wei Guo', Dezhen Qiu', Zhihui Shan', \\ Zhonglu Yang', Songli Yuan', Chanjuan Zhang ${ }^{1}$, Xiaojuan Zhang ${ }^{1}$, Baohui Liu², Fanjiang Kong ${ }^{2}$, Xia Li \\ Xinan Zhou', Lam-Son Phan Tran ${ }^{4,5^{*}}$ and Dong Cao ${ }^{1,2^{*}}$
}

\begin{abstract}
Background: The plant architecture has significant effects on grain yield of various crops, including soybean (Glycine max), but the knowledge on optimization of plant architecture in order to increase yield potential is still limited. Recently, CRISPR/Cas9 system has revolutionized genome editing, and has been widely utilized to edit the genomes of a diverse range of crop plants.

Results: In the present study, we employed the CRISPR/Cas9 system to mutate four genes encoding SQUAMOSA PROMOTER BINDING PROTEIN-LIKE (SPL) transcription factors of the SPL9 family in soybean. These four GmSPL9 genes are negatively regulated by GmmiR156b, a target for the improvement of soybean plant architecture and yields. The soybean Williams 82 was transformed with the binary CRISPR/Cas9 plasmid, assembled with four sgRNA expression cassettes driven by the Arabidopsis thaliana U3 or U6 promoter, targeting different sites of these four SPL9 genes via Agrobacterium tumefaciens-mediated transformation. A 1-bp deletion was detected in one target site of the GmSPL9a and one target site of the GmSPL9b, respectively, by DNA sequencing analysis of two T0-generation plants. T2-generation sp/9a and sp/9b homozygous single mutants exhibited no obvious phenotype changes; but the T2 double homozygous mutant sp/9a/spl9b possessed shorter plastochron length. In T4 generation, higherorder mutant plants carrying various combinations of mutations showed increased node number on the main stem and branch number, consequently increased total node number per plants at different levels. In addition, the expression levels of the examined GMSPL9 genes were higher in the spl9b-1 single mutant than wild-type plants, which might suggest a feedback regulation on the expression of the investigated GmSPL9 genes in soybean.

Conclusions: Our results showed that CRISPR/Cas9-mediated targeted mutagenesis of four GmSPL9 genes in different combinations altered plant architecture in soybean. The findings demonstrated that GmSPL9a, GmSPL9b, GmSPL9c and GmSPL9 function as redundant transcription factors in regulating plant architecture in soybean.
\end{abstract}

Keywords: CRISPR/Cas9, Plastochron length, Plant architecture, Soybean, SPL

\footnotetext{
* Correspondence: son.tran@riken.jp; caodong@caas.cn

${ }^{5}$ Stress Adaptation Research Unit, RIKEN Center for Sustainable Resource

Science, 1-7-22, Suehiro-cho, Tsurumi, Yokohama 230-0045, Japan

${ }^{1}$ Key Laboratory of Biology and Genetic Improvement of Oil Crops, Ministry

of Agriculture, Oil Crops Research Institute, Chinese Academy of Agricultural

Sciences, Wuhan 430062, China

Full list of author information is available at the end of the article
}

(c) The Author(s). 2019 Open Access This article is distributed under the terms of the Creative Commons Attribution 4.0 International License (http://creativecommons.org/licenses/by/4.0/), which permits unrestricted use, distribution, and reproduction in any medium, provided you give appropriate credit to the original author(s) and the source, provide a link to the Creative Commons license, and indicate if changes were made. The Creative Commons Public Domain Dedication waiver (http://creativecommons.org/publicdomain/zero/1.0/) applies to the data made available in this article, unless otherwise stated. 


\section{Background}

Soybean (Glycine max) plant architecture is an important trait for developing high-yield cultivars, and this trait can be determined based on stem growth habit, node number, plant height, internode length, branch number, leaf size and shape $[1,2]$. Previous studies on soybean plant architecture have primarily focused on stem growth habit [1, 3-7]. Recently, Gao et al. (2017) found that the Glycine max INCREASED LEAF PETIOLE ANGLE 1 (GmILPA1), a gene encoding an 'anaphase-promoting complex/cyclosome' $(\mathrm{APC} / \mathrm{C})$ protein, modulated the leaf petiole angle in soybean [8]. In addition, marker-assisted studies have revealed many quantitative trait loci (QTLs) associated with various traits related to plant architecture in soybean, including plant height, internode length, node number, branch number, pod number, and leaflet length and width (http://www.SoyBase.org). However, the molecular mechanisms regulating plant architecture and yield potential remain unknown, and information about the genes responsible for improving soybean plant architecture is still limited.

In plants, most members of the SQUAMOSA PROMOTER BINDING PROTEIN-LIKE (SPL) transcription factor (TF) family are regulated through miR156, and these TFs affect the transition between the juvenile and adult phases [9-12]. In Arabidopsis, SPL9 and SPL15 have been shown to be implicated in the regulation of plastochron length and leaf size $[13,14]$. In rice (Oryza sativa), OsSPL14 has been identified as IDEAL PLANT ARCHITECTURE 1 (IPA1) or WEALTHY FARMER'S PANICLE (WFP) gene, which regulates shoot branching during the vegetative phase and the number of grains produced in a panicle $[15,16]$. The rice OsSPL14 gene encodes the closest homologous protein of the Arabidopsis SPL9 and SPL15, and its overexpression also prolongs plastochron length [17]. The OsSPL14 can directly bind to the promoter of the TEOSINTE BRANCHED 1 (TB1) in rice to suppress rice tillering, and positively and directly regulates the expression of DENSE AND ERECT PANICLE 1 (DEP1) to affect plant height and panicle length [18]. Wang et al. (2015) reported that a spatiotemporally coordinated gene network comprising the miR156/miR529/SPL and miR172/ Apetala2 (AP2) pathways controls tiller and panicle branching in rice [19]. Recently, Wang et al. (2017) identified a RING-finger E3 ligase, named IPA1 INTERACTING PROTEIN 1 (IPI), that can interact with OsSPL14 in the nucleus [20]. IPI1 promotes the degradation of OsSPL14 in panicles, while it stabilizes OsSPL14 in shoot apexes, thereby regulating plant architecture in rice [20]. In soybean, transgenic plants overexpressing the GmmiR156b produced greatly altered plant architecture, leading to a remarkable increase in grain yield per plant [21]. It has also been reported in soybean that the GmSPL9d gene is expressed in the shoot apical meristem (SAM) and axillary meristem (AM), and that GmSPL9d may regulate axillary bud formation and shoot branching by physically interacting with the homeobox protein WUSCHEL (WUS), a central regulator of AM formation [21]. GmmiR156b regulates soybean plant architecture mainly through the direct cleavage of SPL genes [21]. However, our knowledge on the functions of GmSPL9 genes in controlling plant architecture is still limited in soybean.

Recently, the emergence of clustered regularly interspaced short palindromic repeats/CRISPR associated protein 9 (CRISPR/Cas9) technology has brought new opportunities to the field of genetic manipulation in plants [22-24]. It has attracted large attention, and its application has dramatically expanded in genome editing of many crops, including rice [7, 23], wheat (Triticum aestivum) [25-27], maize (Zea mays) [28, 29], oilseed rape (Brassica napus) [30], barley (Hordeum vulgare) [31], cotton (Gossypium hirsutum) [32], tomato (Solanum lycopersicum) [33] and soybean [34-36]. Very recently, Cai et al. (2018) have reported the successful application of the CRISPR/Cas9 system in soybean in mutating the gene Flower Locus $T$ (FT), which resulted in delayed flowering time of mutated plants under both short-day and long-day conditions, suggesting that gene knock-out mediated by the CRISPR/Cas 9 system in soybean research is feasible [36]. However, research in soybean using the CRISPR/Cas9 system is still rare, due to the fact that soybean transformation is still a great challenge for most research groups. Furthermore, most of the targets of the successful applications of the CRISPR/ Cas 9 system in gene editing in soybean were single gene [34-36]. Here, we report the CRISPR/Cas9-based multiple gene editing system to target four SPL9 genes in soybean. T4-generation soybean mutant plants carrying different combinations of mutations exhibited a number of altered characteristics in plant architecture. Our findings indicate that the CRISPR/Cas9 system is a promising tool to advance soybean breeding.

\section{Results}

Target selection and construction of the CRISPR/Cas9 vector system for mutagenesis of four GmSPL genes in soybean

It has been reported that GmmiR156b overexpression improved yield-related phenotypic traits in soybean [21], suggesting the involvement of the GmSPL genes, which are the cleavage targets of GmmiR156b [21], in regulating the architecture of soybean plants in a negative manner. This hint was strengthened by the fact that the GmSPL9a, GmSPL9b, GmSPL9c and GmSPL9d genes were down-regulated in GmmiR156b-overexpressing transgenic soybean plants [21, 37]. Additional file 1: Figure S1 showed that the GmSPL9a, GmSPL9b, GmSPL9c and GmSPL9d were clustered into the 
AtSPL9/AtSPL15 and OsSPL14/OsSPL17 cluster, suggesting that all four GmSPL9 TFs might have a role in altering the architecture of soybean plants. To study their function by a genetic means, three target adaptors, SP1 (selected for targeting GmSPL9a and GmSPL9b genes), SP2 (selected for targeting GmSPL9a and GmSPL9b genes) and SP3 (selected for targeting GmSPL9c and GmSPL9d genes) in the first exon of these four genes, and one target adaptor (SP4) in the second exon of GmSPL9d were chosen for mutagenesis of these four genes in soybean using the CRISPR/Cas9 technology (Fig. 1). The Arabidopsis U3b, U3d, U6-1 and U6-29 promoters were used to drive the individual expression of the 4 sgRNA expression cassettes containing the designed target sites (Fig. 2). These constructs were inserted into the CRISPR/Cas9 vector system designed previously [38] (Fig. 2), and the obtained plasmid was introduced into the soybean Williams 82 variety using Agrobacterium tumefaciens according to the procedure described by Cao et al., 2015 [37].

\section{Targeted mutagenesis of four GmSPL9 genes in soybean} We obtained two T0 transgenic lines with the section for the Bar gene product (Bar-positive). Genomic DNA was extracted from leaves using cetyltrimethyl ammonium bromide (CTAB) to investigate CRISPR/Cas9-induced mutations at the target sites. Sequencing analysis showed that the T0-10 line had a 1-bp deletion in the GmSPL9a-SP1 (Fig. 3a, spl9a allele; Additional file 2: Table S1), while the T0-20 line had a 1-bp deletion in the GmSPL9b-SP1 (Fig. 3b, spl9b-1 allele; Additional file 2: Table S1; Additional file 3: Figure S2A), resulting in frame-shift mutations in both GmSPL9a and GmSPL9b genes (Additional file 2: Table S1; Additional file 3: Figure S2A). Both these two mutations generated premature translation termination codons (PTCs), and thus are null mutations (Additional file 3: Figure S2A; Additional file 4: Text S1). However, we found that the other five target sites GmSPL9a-SP2, GmSPL9b-SP2, GmSPL9c-SP3, GmSPL9d-SP3 and GmSPL9d-SP4 showed no edited mutations in both two T0 plants. Subsequently, we analyzed four T1-10 plants and six T1-20 plants and found two new edited types; one in the target site GmSPL9b-SP1 (39-bp deletion) (Fig. 3c, spl9b-2 allele; Additional file 2: Table S1; Additional file 3: Figure S2A) and another in the target site GmSPL9c-SP3 (6-bp deletion) (Fig. 3d, spl9c allele; Additional file 2: Table S1; Additional file 3: Figure S2B). The 39-bp deletion resulted in a 12-amino-acid deletion (from position 28 to 39 ) and an amino-acid substitution (F40 V) in the GmSPL9b protein (Additional file 3: Figure S2A), while the 6-bp deletion

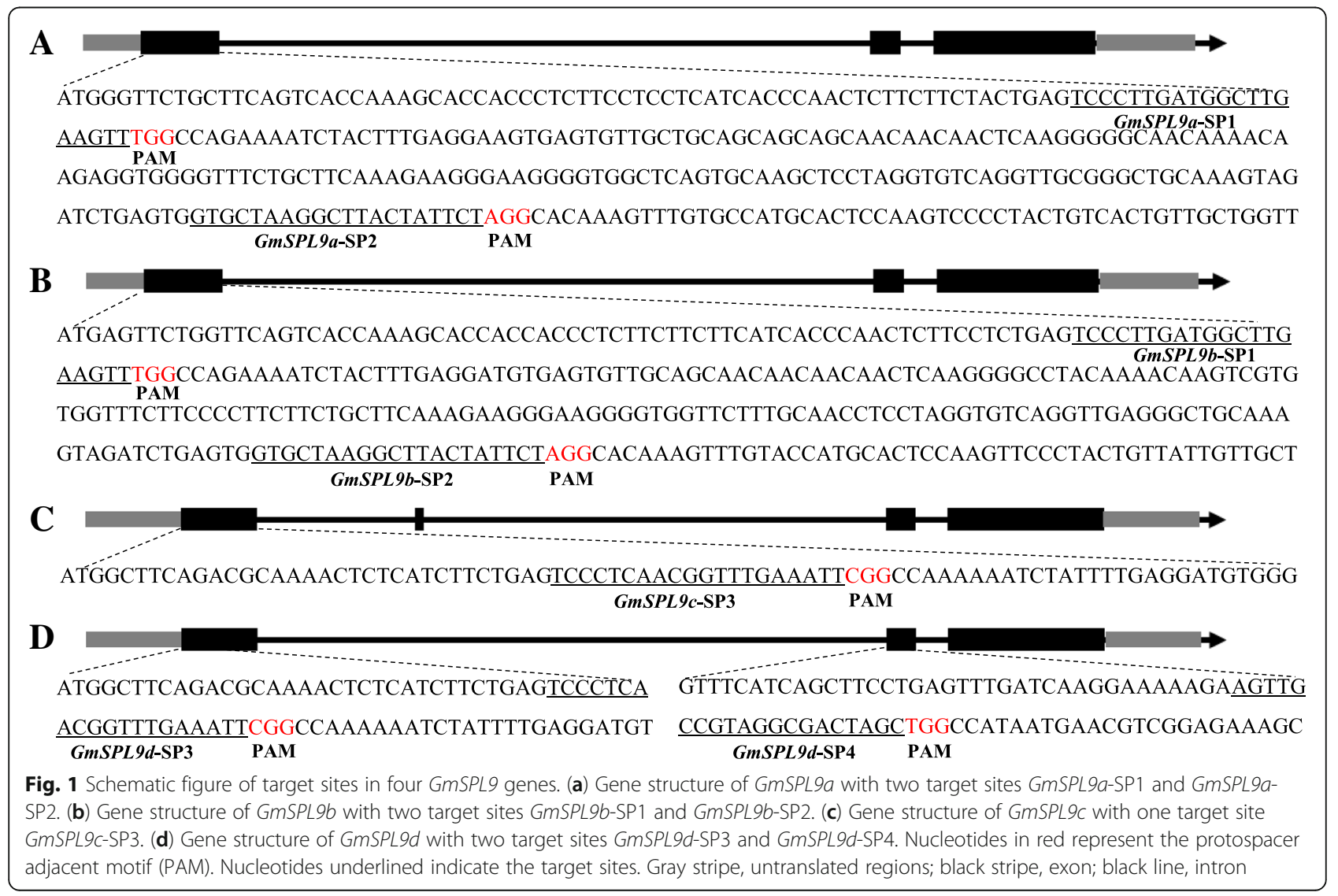




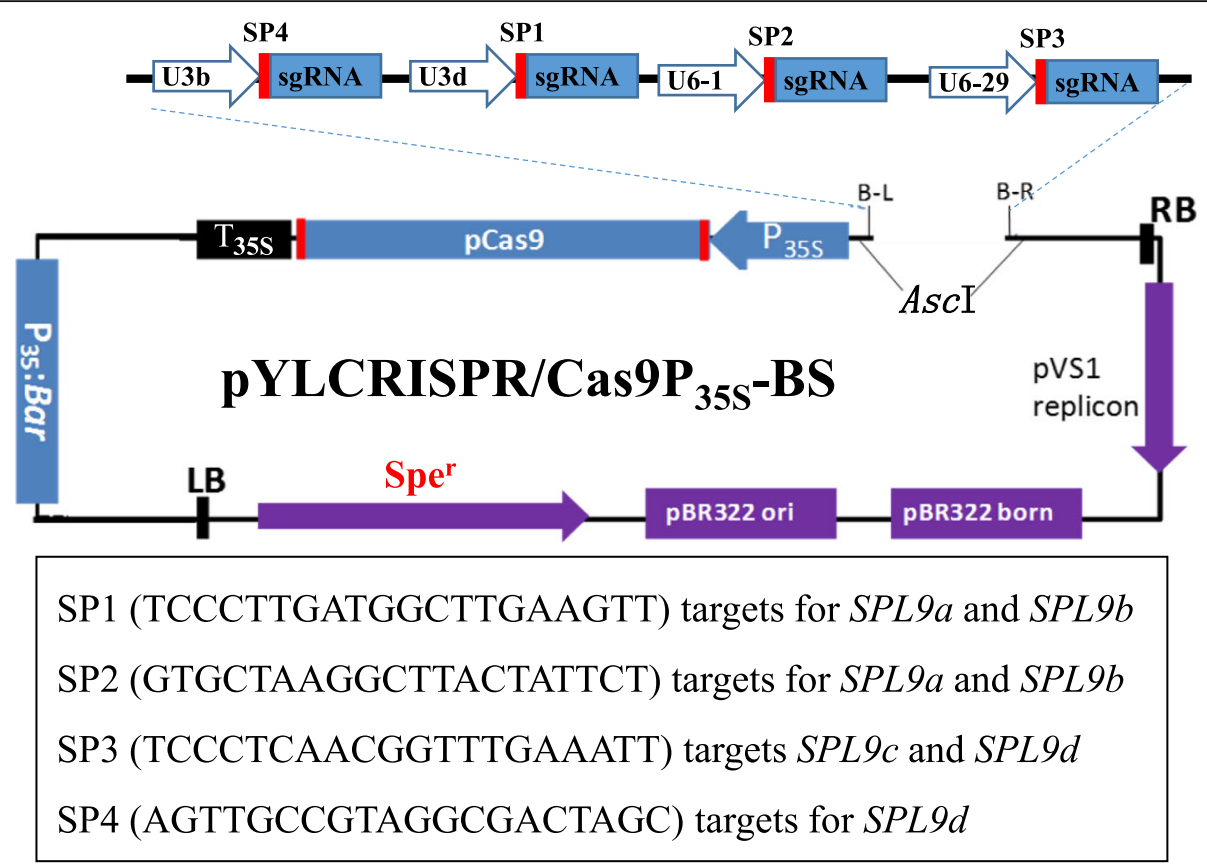

Fig. 2 Schematic figure of the binary vector designed for mutagenesis of the four GmSPL9 genes using the CRISPR/Cas9 technology. The pYLCRISPR/Cas9P ${ }_{355}$-BS was derived from the pYLCRISPR/Cas9P ${ }_{355}$-B [38]. The target adaptor SP1, targeting two sites (GmSPL9a-SP1 and GmSPL9bSP1), directed by the Arabidopsis thaliana U3d promoter; the target adaptor SP2, targeting two sites (GmSPL9a-SP2 and GmSPL9b-SP2), directed by the A. thaliana U6-1 promoter; the target adaptor SP3, targeting two sites (GmSPL9C-SP3 and GmSPL9d-SP3), directed by the A. thaliana U6-29 promoter; the target adaptor SP4, targeting one site (GMSPL9d-SP4), directed by the A. thaliana U3b promoter

caused a deletion of two amino acids in the GmSPL9c protein (from position 16 to 17) (Additional file 3: Figure S2B).

There is currently not much knowledge with respect to the functions of GmSPL9a, GmSPL9b and GmSPL9c on the regulation of plant architecture. However, there was a report about the function of GmSPL9d with regard to its regulatory function in plant architecture. Specifically, overexpression of the GmSPL9d gene suppressed the branch number in Arabidopsis transgenic plants [21]. Thus, given the redundant functions of the GmSPL9 genes, to obtain profound evidence for their genetic involvement in regulating plant architecture, we were interested in identifying the higher-order mutants, especially those that contain mutation in the GmSPL9d gene (Fig. 3e; Additional file 2: Table S1). The seeds of ten T1-generation plants (four T1-10 plants and six T1-20 plants) were sown, and the DNAs of 120 independent T2 plants (12 independent T2 plants from each T1-generation plant) were obtained. We then mixed the DNAs of 12 independent T2 plants from each T1-generation plant as one pooled DNA template for PCR, resulting in 10 DNA pools. Sequence analysis showed that there were no edited mutations in the two target sites of GmSPL9d (GmSPL9d-SP3 and GmSPL9d-SP4) among the examined T2 plants. When we obtained the T3-generation seeds, we conducted similar experiments to identify spl9d mutants. The pooled DNAs of T3-10-1-3 (mixed 12 plants) and T3-10-1-6 (mixed 12 plants) had a new edited type in GmSPL9d (spl9d allele, Additional file 2: Table S1). Further sequence analysis showed a 1-bp deletion in the target site of GmSPL9d-SP3 in T3-10-1-3-2 (Fig. 3e; Additional file 2: Table S1), resulting in the spl9a (-/-)/spl9b-1 (-/-)/spl9d (+/-) mutant. In addition, we also obtained a 'transgene-clean' spl9b-1 (-/-) mutant line from the T2-10-1-1 line using the selectable marker gene Bar for selection (Additional file 2: Table S1; Additional file 5: Figure S3). The T2-10-1-1 line had 1-bp deletion in the target site of GmSPL9b-SP1, and its T3 and T4-generation plants were all 'transgene-clean' homozygous spl9b-1 mutants. After four generations of selection, we obtained the 'transgene-clean' homozygous spl9b-1 single and the $s p l 9 a / s p l 9 b-1 / s p l 9 c / s p l 9 d$ homozygous quadruple mutants, and some other mutants that were still Bar-positive like spl9a (-/-)/spl9b-1 (-/-), spl9a (-/-)/spl9b-1 $(-/-) / \operatorname{spl} 9 c(+/-), \operatorname{spl} 9 a(-/-) / s p l 9 b-2(-/-) / s p l 9 c(+/-)$ and spl9a (-/-)/spl9b-1 (-/-)/spl9c (+/-)/spl9d (+/-) from our genome editing experiment (Additional file 2: Table S1).

\section{The examined four SPL9 genes regulate soybean plant architecture}

In the $\mathrm{T} 1$ and $\mathrm{T} 2$ generations, the obtained single mutants $s p l 9 b-1$ and $s p l 9 c$ showed no differences in node number on main stem as compared with wild-type (WT) plants, whereas the obtained $s p l 9 a / s p l 9 b-1$ double mutant plants had one more trifoliate leaf than the WT 
A w82: ICccttgatgGctTGahgtTtgG T0-10: TCCCTTGATGGCTIG - AGTITGG - 1bp TCCCT TGATGGCTTGAG TTTGGCCAG

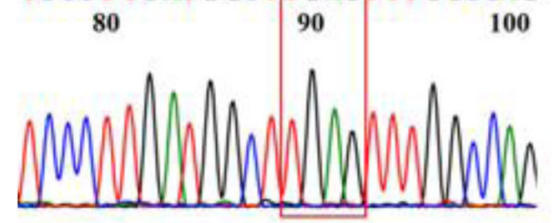

B W82: CCAACTTCAGGCCATCAAGGGA T0-20: CCAAA - TTCAAGCCATCAAGGGA - 1bp

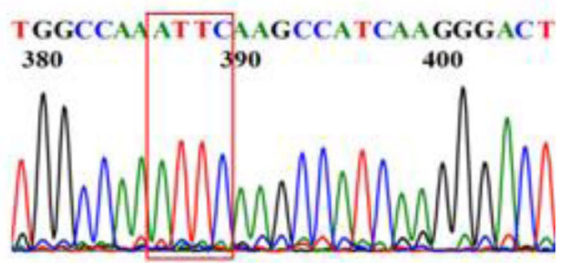

C W82: GTTGTTGTTGCTGCANCACTCACATCCTCAMGTAGATTTTCTGGCCAMCTTCMGCCATCAMGGA T1-10-3: GTTGTTGTTGCTGCAA …. .

\section{GTT GTT GT TGTT GC TGCAAAGCCATCAAGGGA}

340

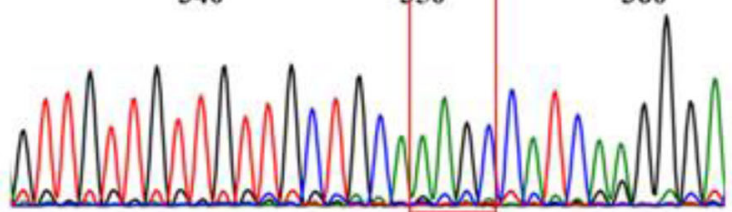

\section{D}

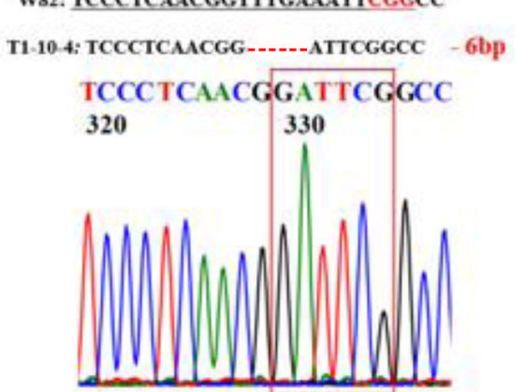

E W22: CCGAATTICAMACCGTTGAGGGA T3-10-1-3-2: CCGATT-CAMCCGTTGAGGGA - Ibp

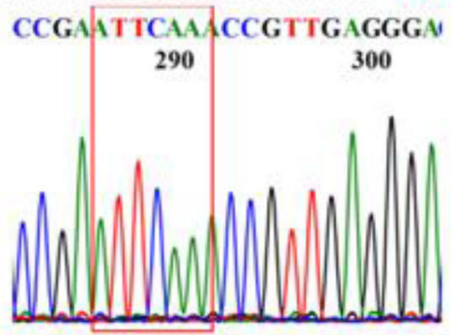

Fig. 3 Results obtained from mutagenesis of four GmSPL9 genes by CRISPR/Cas9 technology. (a) Detailed sequence of the target site GmSPL9aSP1 in the T0-10 line. (b) Detailed sequence of the target site GmSPL9b-SP1 in the T0-20 line. (c) Detailed sequence of the target site GmSPL9bSP1 in the T1-10-3 line. (d) Detailed sequence of the target site GmSPL9C-SP3 in the T1-10-4 line. (e) Detailed sequence of the target site GmSPL9d-SP1 in the T3-10-1-3-2 line. Nucleotides in red and underlined represent the protospacer adjacent motif (PAM). The underlined nucleotides indicated the target sites. '-' signs indicate the number of deleted nucleotides. W82 represents Williams 82 wild-type sequence

when they were grown in an artificial climate chamber at 12-h light/12-h dark photoperiod and $24{ }^{\circ} \mathrm{C}$ (Additional file 6: Figure S4a). When we were growing the different T4 mutant plants under artificial climate chamber at 15 -h light/9-h dark photoperiod and $28^{\circ} \mathrm{C}$, we found that they exhibited notable changes in plant architecture (Fig. 4). For instance, similar to transgenic plants overexpressing GmmiR156b (GmmiR156b-OX), the T4 spl9a/spl9b-1/ spl9c/spl9d homozygous quadruple mutant plants showed more branches than WT, including some secondary branches originated from the primary branches (Fig. 4a). The T4 'transgene-clean' homozygous spl9b-1 single mutant plants showed no difference in node number on main stem (Fig. 4b), which was in agreement with the results obtained in the T1- and T2-generation spl9b-1 (-/-) mutant plants, but the spl9b-1 single mutant plants exhibited 15.5 and $33.0 \%$ increase in total node number per plant and branch number, respectively, as compared with that of WT plants (Fig. 4c-d). Furthermore, the T4 spl9a (-/-)/spl9b-1 (-/-)/spl9c (+/-) and spl9a $(-/-) / \operatorname{spl} 9 b-2(-/-) / \operatorname{spl} 9 c(+/-)$ mutant plants showed 16.3 and $7.7 \%$ increase in node number on main stem, 73.7 and $36.3 \%$ increase in total node number per plant, 72.5 and $57.8 \%$ increase in branch number, and 52.2 and $15.2 \%$ increase in dry weight, respectively, relative to that of WT plants (Fig. $4 \mathrm{~b}-\mathrm{e}$ ). Interestingly, the T4 spl9a (-/-)/spl9b-1 (-/-)/spl9c (+/-) mutant plants exhibited more remarkable phenotypic changes in the parameters examined compared with the T4 spl9a (-/-)/spl9b-2 (-/-)/spl9c (+/-) mutant plants (Fig. 4b-e), suggesting the mutation in spl9b-1 was more severe than that in $s p l 9 b-2$. Additionally, the T4 spl9a (-/-)/spl9b-1 (-/-)/spl9c (+/-)/spl9d $(+/-)$ and spl9a (-/-)/spl9b-1 (-/-)/spl9c (-/-)/spl9d $(-/-)$ mutant plants, when analyzed together, generally displayed the most significant changes in plant architecture when comparing with WT and the lower-order mutants, showing the highest node number on main stem, branch number, total node number 
A

WT

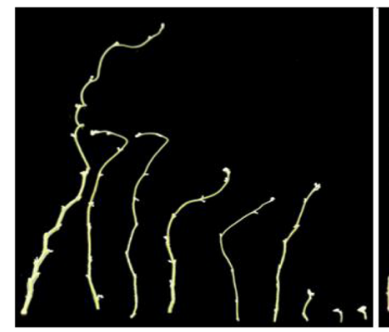

GmmiR156b-OX

spl9a/spl9b-1/spl9c/spl9d

B

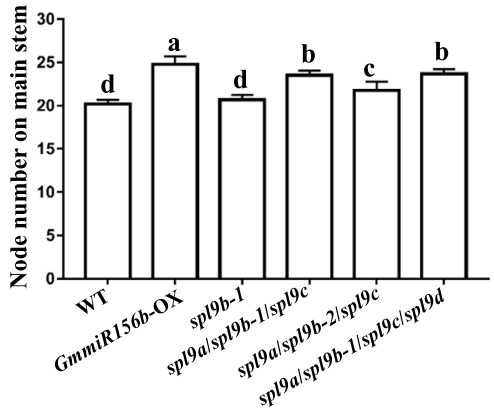

D

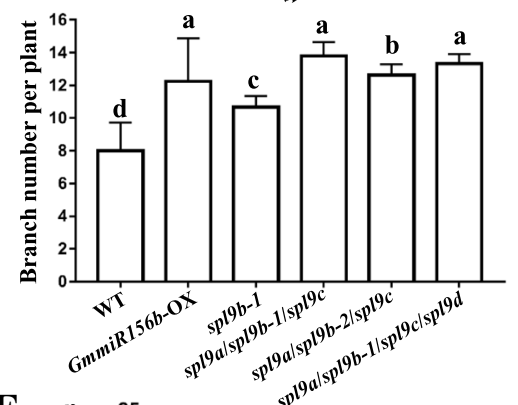

C

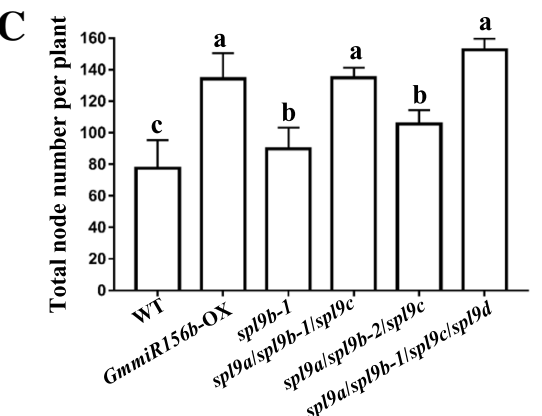

$\mathbf{E}$

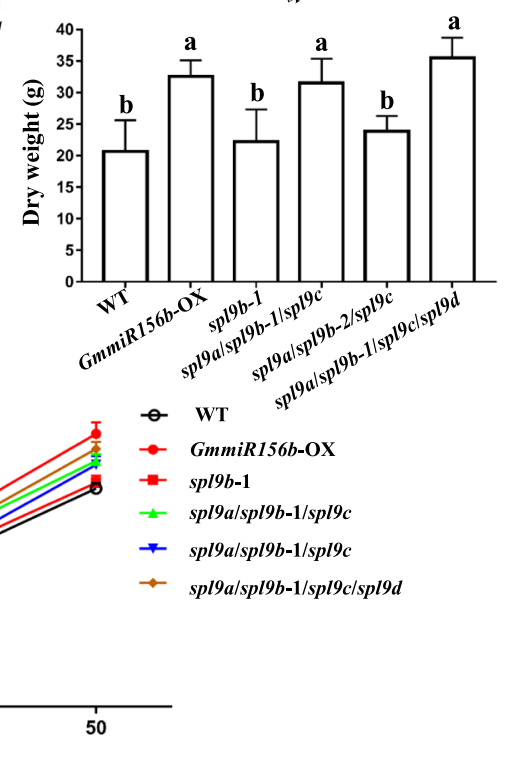

Fig. 4 The soybean CRISPR/Cas9-induced T4-generation sp/9 mutant lines showed altered plant architecture. (a) Representative pictures showing the primary and secondary branches of wild-type (WT), GmmiR156b-overexpressing (GmmiR156b-OX) and spl9a (-/-)/spl9b-1 (-/-)/spl9c $(-/-) /$ spl9d (-/-) quadruple mutant plants at 50th day after emergence, after the leaves were removed. Red arrows indicate the secondary branches on primary branches. (b) Node number on main stem, (c) total node number per plant, (d) branch number, and (e) dry weight were recorded after the experiment was completed at day 50th after emergence. (f) Dynamic changes in the trifoliate leaf number on main stem in different genotypes. (b-f) The data of WT, GmmiR156b-OX and the spl9b-1 (-/-) single mutant plants were obtained from six plants. The data of sp/9a/spl9b-1/spl9c were obtained from five sp/9a (-/-)/spl9b-1 (-/-)/sp/9c (+/-) plants, the data of sp/9a/spl9b-2/spl9c were obtained from nine spl9a (-/-)/spl9b-2 (-/-)/spl9c (+/-) plants, and the data of spl9a/spl9b-1/spl9c/spl9d were obtained from two spl9a (-/-)/spl9b-1 (-/-)/spl9c $(-/-) /$ spl9d (-/-) and one spl9a (-/-)/spl9b-1 (-/-)/spl9c (+/-)/spl9d (+/-) plants. "a, b, c and d" indicate statistically significant differences among the genotypes (one-way ANOVA of variance, $P<0.05$ )

per plant and dry weight among the examined genotypes (Fig. 4b-e). It is worth noting that these highest-order mutant plants, when analyzed together, showed a $13.2 \%$ increase in total node number per plant and a $12.6 \%$ increase in dry weight (Fig. 4c and e), but similar node number on main stem and branch number (Fig. 4b and d), in comparison with the T4 spl9a (-/-)/spl9b-1 (-/-)/spl9c (+/-) mutant plants. Taken together, our data indicated that all four SPL9 genes have important roles in regulating soybean plant architecture, both redundantly and independently. 
The examined four SPL9 genes regulate plastochron length in soybean

The T4-generation spl9b-1 single homozygous mutant showed no difference in number of trifoliates compared with WT, whereas the other T4 higher-order mutant plants examined showed shorter plastochron lengths in soybean compared with the spl9b-1 single mutant and WT plants (Fig. 4f). In particular, 50 days after emergence (DAE), WT, GmmiR156b-OX, spl9b-1, spl9a $(-/-) /$ spl $9 b-1(-/-) / s p l 9 c(+/-)$ and spl9a (-/-)/spl $9 b-2$ $(-/-) / \operatorname{spl} 9 c(+/-)$ exhibited trifoliate leaves on the main stem in an average number of 18.2, 22.8, 18.7, 20.5 and 20.2, respectively, while the spl9a (-/-)/spl9b-1 $(-/-) / s p l 9 c(+/-) / s p l 9 d(+/-)$ and spl9a (-/-)/spl9b-1 $(-/-) / \operatorname{spl} l 9 c(-/-) /$ spl9d $(-/-)$ mutant plants, when analyzed together, had the average number of trifoliate leaves of 21.5 (Fig. 4f). In addition, both the T2-generation spl9a (-/-)/spl9b-1 (-/-) double mutant and GmmiR156$b$-OX plants had shorter plastochron lengths compared with WT plants (Additional file 6: Figure S4). These data indicated that these four GmSPL9 genes are implicated in regulation of plastochron length in soybean, perhaps under the control of GmmiR156b.

GmSPL9b regulates expression of the four GmSPL9 genes To validate the effect of the mutation in GmSPL9b gene (spl9b-1 allele) on the expression of the four GmSPL9 genes in soybean, we analyzed the expression levels of all four GmSPL9 genes in leaves and SAM of the stable spl9b-1 (Bar negative) single mutant and WT plants grown under artificial climate chamber (15-h light/9-h dark) at 20th DAE using quantitative RT-PCR (qRT-PCR). Figure 5 showed that the transcript levels of all four GmSPL9 genes were higher in both SAM and leaves of the $s p l 9 b-1$ single mutant than in that of WT plants. To further validate whether the GmSPL9b TF regulates the expression of GmSPL9a, GmSPL9c and GmSPL9d genes in soybean, we generated transgenic lines overexpressing the GmSPL9b. The two independent transgenic lines displayed higher expression levels of the GmSPL9b gene in leaves than WT (Additional file 7: Figure S5A). Furthermore, our data revealed that the transgenic plants, especially line \#5 with higher expression level of $G m S P L 9 b$, had lower transcript levels of GmSPL9a, GmSPL9c and GmSPL9d than WT plants (Additional file 7: Figure S5B-D). However, similar to the spl9b-1 single mutant plants, the transgenic lines overexpressing GmSPL9b showed comparable plant architecture as WT plants as evidenced by the data of node number on main stem, total node number per plant and branch number per plant (Additional file 7: Figure S5E-G). Taken together, these results indicated that GmSPL9b gene may repress the expression of GmSPL9a, GmSPL9c and GmSPL9d, as well as itself in soybean.

\section{Discussion \\ CRISPR/Cas9 genome editing efficiency in soybean - single construct for multiple mutations}

In the current study, we explored the CRISPR/Cas9 system for mutagenesis of four GmSPL9 genes by using a single plasmid construct in an attempt to make loss-of-function soybean mutants to assess the functions of these genes in regulation of plant architecture (Figs. 1-2). We designed four target adaptors (SP1, SP2, SP3 and SP4) and found that only the SP1 adaptor targeting GmSPL9a and GmSPL9b genes produced heterozygous mutants in
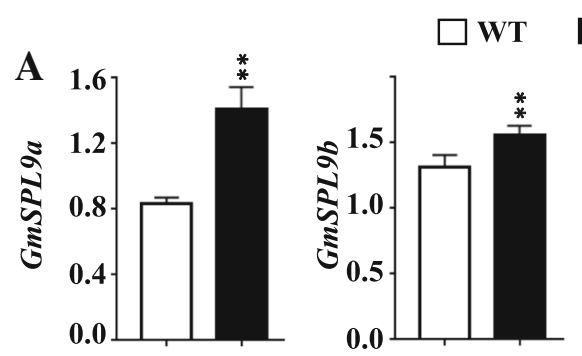

spl9b-1
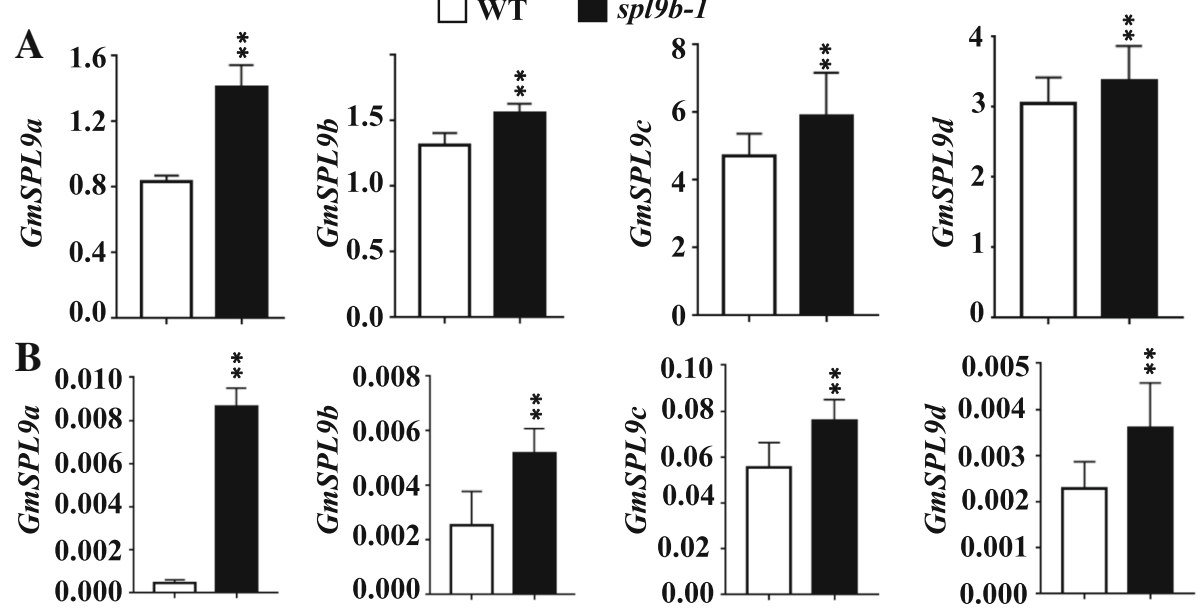

Fig. 5 Expression patterns of four GMSPL9 genes in wild-type (WT) and the spl9b-1 (Bar negative) mutant plants. (a) Relative expression of GmSPL9a, GmSPL9b, GmSPL9c and GmSPL9d in shoot apical meristem of WT and spl9b-1 plants. (B) Relative expression of GmSPL9a, GmSPL9b, GMSPL9c and GmSPL9d in leaves of WT and spl9b-1 plants. The plants were grown under artificial climate chamber (15-h light/9-h dark) conditions for 20 days after emergence. Relative transcript levels were assessed by quantitative RT-PCR and normalization to the expression level of the GMTUB gene. Expression levels shown are means \pm SEs of three replicates (** $P<0.01$; Student's $t$-test) 
T0-generation plants (Fig. 1; Additional file 2: Table S1). However, the editing continued to work with the constructed CRISPR/Cas9 system in plants of later generations (Bar-positive), and new mutants were obtained at different target sites (Additional file 2: Table S1). Our data indicated that the genome editing efficiency was low in T0-generation of soybean, but relatively high in T1-generation plants (Additional file 2: Table S1). Similar results were reported in Arabidopsis, which showed that the editing efficiency and editing types induced by CRISPR/ Cas9 were relatively low, and both uniform and chimeric mutations were occurred in the T1-generation [38-40]. It has been suggested that Arabidopsis transformation methods using vegetative tissues might produce relatively low editing efficiency [38]. Thus, the low editing efficiency in the soybean T0-generation observed in this study might also be caused by our transformation method using cotyledonary node. Furthermore, we observed that the SP2 and SP4 adaptors designed for three target sites (GmSPL9 $a$ and GmSPL9b genes, and GmSPL9d gene, respectively) showed no edits in T0-, T1-, T2-, T3- and T4-generation plants. Previous studies reported that selection of target sequences with relatively higher GC content might result in a higher editing efficiency [38]. We should select target sites with higher GC content to improve the editing efficiency. Therefore, at least in the case of soybean, a crop with low transformation frequency [41], less target genes with more target sites in each target gene should be designed to make sure that lower-order mutants can be generated. Subsequently, higher-order mutants can be obtained through crossing.

\section{Functions of the four examined GmSPL9 genes in regulating plant architecture of soybean}

In rice, many studies have reported that the OSSPL14 had a great role in regulation of plant architecture [1520]. In Arabidopsis, SPL9, SPL15 and SPL10 function in a redundant manner to regulate plastochron length [13, 14]. In bread wheat, miR156 was reported to control plant architecture via the repression of a group of $S P L$ genes [42]. The SPL TFs share a highly conserved DNA-binding domain called SQUAMOSA PROMOTER BINDING PROTEIN (SBP)-box [42]. Several maize SBP-box-type TFs, such as TEOSINTE GLUME ARCHITECTURE (TGA1) [43], TASSELSHEATH4 (TSH4) [44], UNBRANCHED2 (Ub2) and UNBRANCHED3 (Ub3) [45], were shown to be associated with maize grain architecture. In soybean, a previous study reported that overexpression of GmmiR156b improved plant architecture, and consequently grain yield [21]. Ectopic overexpression of GmSPL9d reduced branch number in Arabidopsis [21]. However, the functions of GmSPL9d and its closest homologs, like GmSPL9a, GmSPL9b and
GmSPL9c TFs (Additional file 1: Figure S1), in influencing soybean plant architecture remained to be determined.

Using the CRISPR/Cas9 system for genome editing, we were able to produce a number of single and higher-order mutants, particularly the quadruple mutant, for investigating the roles of these four TFs in forming soybean plant architecture. Specifically, we obtained the homozygous mutants spl9b-1 and spl9al spl9b-1/spl9c/spl9d after 4 generations (Additional file 2: Table S1). Detailed analyses of the mutants in different generations showed that the T4 higher-order mutants carrying various combinations of mutations exhibited various increased levels in node number on main stem, total node number per plant, branch number and dry weight compared with WT and spl9b single mutant plants (Fig. 4). Our results suggest that these four GmSPL9 TFs might very likely regulate these characteristics of plant architecture in soybean. All lower- and higher-order mutants in all combinations of these four GmSPL9 genes should be obtained to clearly classify the important level of each of these four TFs in formation of soybean plant architecture. A new genome editing design is required to fulfill this task.

As discussed previously, we just obtained one $s p l 9 b$ single mutant plants with Bar negative, but did not obtained other three single mutants with individual mutation in GmSPL9a, GmSPL9c and GmSPL9d genes in order to clearly classify the functional roles of these four TFs. Thus, we could use only this stable spl9b single mutant as an example to analyze the expression patterns of all four GmSPL9 genes. Our results showed that the $s p l 9 b$ single mutant plants had higher expression levels of all four GmSPL9 genes in both SAM and leaves than WT (Fig. 5), while the transgenic plants overexpressing GmSPL9b displayed lower expression levels of GmSPL9a, GmSPL9c and GmSPL9d genes than WT plants (Additional file 7: Figure S5). This finding suggested that the GmSPL9b TF might negatively regulate the expression of GmSPL9 genes in soybean, which might result in no or minor changes in the spl9b single mutant, when compared with WT plants, with respect to the examined phenotypic parameters (Fig. 4). Further studies need to be conducted using all combinations of single, double, triple and quadruple mutant plants of these four GmSPL9 genes to examine their complex functions in soybean plant architecture and the feedback mechanism underlying the expression patterns of the examined GmSPL9 genes. In addition, our data were obtained under artificial climate chamber conditions. Further studies under field conditions are required to reveal the roles of these four GmSPL9 genes in regulating soybean plant architecture and especially grain yield, prior to using them in genetic engineering for improvement of soybean productivity. 


\section{Conclusions}

The CRISPR/Cas9 system currently has become a versatile tool to advance crop plant breeding. In our present study, we used CRISPR/Cas9-based multiple genome editing, and successfully obtained several mutants, including the quadruple mutant, for assessment of the functions of four closely homologous GmSPL9 genes in formation of soybean plant architecture. Using these mutants, we found that the four GmSPL9 genes may have redundant or independent roles in regulating soybean plant architecture, depending on the phenotypic trait(s) examined. Our data also suggested that the GmSPL9b gene can regulate the expression of the four GmSPL9 genes, including itself, in soybean. Taken together, results of our studies improve the understanding of the application of CRISPR/Cas9 system and provide more knowledge on the regulation of plant architecture in soybean.

\section{Methods}

\section{Plant materials and growth conditions}

The soybean [Glycine $\max$ (L.) Merr.] cultivar Williams 82 was used for transformation. The WT plants, GmmiR156b-overexpressing transgenic plants (line \#5 from Sun et al., 2018) [21], and mutant plants were cultivated in an artificial climate chamber under the conditions of 12 -h light and 12-h dark photoperiod at $24{ }^{\circ} \mathrm{C}$.

To investigate the plant architecture of transgenic plants, 17 T4-generation higher-order spl9 mutant plants of different combinations (Fig. 4) were grown in an artificial climate chamber under the 15-h light/9-h dark photoperiodic conditions at $28^{\circ} \mathrm{C}$. The WT, GmmiR156b-overexpressing transgenic and the homozygous spl9b-1 single mutant (Bar negative) plants were grown with 6 seedlings/each genotype (Fig. 4). The seeds of each genotype were germinated on moistened filter paper for 4 days at $28{ }^{\circ} \mathrm{C}$ and $60 \%$ humidity under 15 -h light/9-h dark photoperiodic conditions. Germinated seedlings were transferred into $25 \mathrm{~cm} \times 25 \mathrm{~cm}$ pot with each pot containing one seedling. All examined phenotypic parameters, including branch number (first branch number on main stem), node number on main stem and total node number per plant, were recorded at 50 DAE. To investigate the plastochron length in the T4 soybean mutants, the trifoliate leaves on main stem were recorded every 10 DAE from 20 to 50 DAE.

\section{Construction of phylogenetic tree}

The full-length protein sequences of 43 soybean SPLs were retrieved from the Phytozome (www. Phytozome. net/) and used to construct a phylogenetic tree to study the relationships of the soybean SPLs with the AtSPL9, AtSPL15, OsSPL14 and OsSPL17 proteins, whose full-length protein sequences were also downloaded from Phytozome. MEGA v.7.0 was used to construct the Neighbour-Joining unrooted tree [46].

\section{Plasmid construction and soybean transformation}

The nucleotide sequence of the four GmSPL genes were downloaded from Phytozome. The target sequence adaptors were designed using the web tool CRISPR-P (http://cbi.hzau.edu.cn/crispr/). The kanamycin resistance gene in the pYLCRISPR/Cas $9 \mathrm{P}_{35 \mathrm{~s}}-\mathrm{B}$, which was received from $\mathrm{Ma}$ et al. [38], was replaced by the spectinomycin resistance $\operatorname{aad} A$ gene, resulting in the pYLCRISPR/Cas $9 \mathrm{P}_{35 \mathrm{~S}}$-BS. The four target sequence adaptors were integrated into different sgRNA expression cassettes and built into the pYLCRISPR/ Cas9P $35 \mathrm{~S}-\mathrm{BS}$ vector according to the protocol reported by $\mathrm{Ma}$ et al. (2015) [38]. Briefly, a digestion/ligation reaction for each sgRNA expression cassette was prepared as follows: $1 \mu \mathrm{L} 10 \times$ CutSmart buffer, $20 \mathrm{ng}$ pYLsgRNA plasmid DNA, $0.5 \mu \mathrm{L}$ target adapter, $3 \mathrm{U}$ BsaI-HF, $20 \mathrm{U}$ T4 DNA ligase, $0.5 \mu \mathrm{L} 10 \times$ NEB T4 DNA ligase buffer and deionized $\mathrm{H}_{2} \mathrm{O}$ to a final volume of $10 \mu \mathrm{L}$. Subsequently, the digestion/ligation reaction was performed in a thermal cycler without using a heated lid at the following thermal cycling program: 10 cycles of $5 \mathrm{~min}$ at $37^{\circ} \mathrm{C}$ and $5 \mathrm{~min}$ at $20^{\circ} \mathrm{C}$. Next, all four sgRNA cassettes were amplified by PCR using the products of digestion/ ligation reaction as template and the site-specific primer pairs (Additional file 8: Table S2) at the following thermal cycling program: 22 cycles of $10 \mathrm{~s}$ at $95^{\circ} \mathrm{C}, 15 \mathrm{~s}$ at $58^{\circ} \mathrm{C}$ and $20 \mathrm{~s}$ at $68^{\circ} \mathrm{C}$. Equal amounts of the obtained PCR products were mixed and purified using a PCR product purification kit (Axygen, California, USA). Finally, a digestion/ligation reaction was prepared to assemble the four sgRNA cassettes into the pYLCRISPR/ Cas9P $_{35 \mathrm{~S}}$-BS as follows: $1.5 \mu \mathrm{L} 10 \times$ CutSmart buffer, $100 \mathrm{ng}$ pYLCRISPR/Cas9P $35 \mathrm{~s}-\mathrm{BS}$ plasmid DNA, $100 \mathrm{ng}$ pooled sgRNA cassettes (the mixture of four PCR products obtained from the previous step), $10 \mathrm{U} \mathrm{BsaI-HF,} 40$ U T4 DNA ligase, $0.5 \mu \mathrm{L} 10 \times$ NEB T4 DNA ligase buffer and deionized $\mathrm{H}_{2} \mathrm{O}$ to a final volume of $15 \mu \mathrm{L}$. Subsequently, the digestion/ligation reaction was performed in a thermal cycler without using a heated lid at the following thermal cycling program: 15 cycles of $5 \mathrm{~min}$ at $37^{\circ} \mathrm{C}$, $5 \mathrm{~min}$ at $10^{\circ} \mathrm{C}$ and $5 \mathrm{~min}$ at $20^{\circ} \mathrm{C}$.

The obtained CRISPR/Cas9 plasmid carrying the sgRNA cassettes was transformed into A. tumefaciens strain EHA105, followed by the soybean transformation that was conducted according to the description previously reported by Cao et al., 2015 [37] with some modifications. Briefly, sterilized Williams 82 seeds were germinated in B5 medium for one day, and then the one-day-old germinated seedlings were vertically cut at cotyledonary node, and any remaining axial shoot/bud parts attached to the cotyledonary node were removed. 
Subsequently, the explants were wounded with a scalpel and dipped into the A. tumefaciens strain EHA105 carrying the CRISPR/Cas9 plasmid with the sgRNA cassettes built-in. After 4 days of co-cultivation in co-cultivation medium, the explants were transferred into the shoot induction medium without glufosinate. Seven days later, the explants were transferred into the shoot induction medium with $10 \mathrm{mg} \mathrm{L}^{-1}$ glufosinate for 2 weeks. Subsequently, the explants were cultured in the shoot elongation medium containing $5 \mathrm{mg} \mathrm{L}^{-1}$ glufosinate. When the elongated shoots were about $3 \mathrm{~cm}$, they were transferred to rooting medium without further selection. Glufosinate $\left(160 \mathrm{mg} \mathrm{L}^{-1}\right)$ was applied until the first trifoliate appeared to screen for T0, T1 and T2 transformants.

To obtain transgenic soybean plants overexpressing $G m S P L 9 b$, the GmSPL9b-pTF101 vector harboring the GmSPL9b gene under the $35 S$ promoter from cauliflower mosaic virus was used to transform the soybean cultivar Dongnong 50, according to the description previously reported by Cao et al., 2015 [37].

\section{DNA extraction and mutation screening}

Genomic DNA was extracted from the leaves of each independent T0, T1, T2, T3 and T4 plant and used for PCR. The target site sequences were amplified by PCR with sequence-specific primer sets (Additional file 8: Table S2), and the PCR products were then separated by electrophoresis on $1.0 \%$ agarose in $1 \times$ TAE buffer. The purified DNA fragments were sequenced and analyzed. The successfully edited types could be identified via sequence peaks and alignment to the reference sequences. The heterozygous mutants showed overlapping peaks near the target site, and the homozygous mutants were identified by sequence alignment with the WT sequence.

To screen the spl9d mutants in T2 generation, the seeds obtained from 10 T1-generation plants (four T110 plants and six T1-20 plants) were sown to sample DNAs from 120 independent T2 plants (12 independent T2 plants from each T1-generation plant). The DNAs of 12 independent $\mathrm{T} 2$ plants from each T1-generation plant were then pooled as a DNA template for PCR, resulting in a total of 10 DNA pools. To screen the spl9d mutants in T3 generation, similar protocol was conducted as described above in T2 generation. To screen the spl9d mutants in T4 generation, the DNA of every independent T4 plant was used as template for PCR. The PCR products were digested with EcoRI (New England Biolabs) and then separated by $1.0 \%$ agarose in $1 \times$ TAE buffer.

\section{RNA isolation, CDNA synthesis and qRT-PCR analysis}

The shoot apical meristem (SAM) and third fully developed trifoliate leaves from the bottom of the plants were sampled at 20th DAE and immediately frozen in liquid nitrogen. Total RNA was isolated from each sample using TRIzol reagent (Invitrogen, USA), and cDNA was synthesized for quantitative RT-PCR (qRT-PCR) to assess the transcript levels of GmSPL9a (Glyma.02G177500), GmSPL9b (Glyma.09G113800), GmSPL9c (Glyma.03G143100), GmSPL9d (Glyma.19G146000) and GmTUB (Glyma.08G014200) (as an internal control) as described previously in Cao et al., (2015) [37]. The primers used for qRT-PCR are listed in Additional file 8: Table S2. The qRT-PCR mixture was prepared by mixing $1 \mu \mathrm{L}$ of the cDNA synthesis reaction mixture with $2.5 \mu \mathrm{L}$ forward primer (final concentration $1.0 \mathrm{mM}$ ), $2.5 \mu \mathrm{L}$ reverse primer (final concentration $1.0 \mathrm{mM}$ ), $10 \mu \mathrm{L}$ of SYBR Premix Extaq Perfect Real Time (TAKARA Bio Inc., Japan) and water to a final volume of $20 \mu \mathrm{L}$. The qRT-PCR was performed using the program as essentially described in Nan et al. (2014) [47].

\section{Data analysis}

Data of phenotype were analyzed with the SPSS (Version 21.0) using one-way analysis of variance. Data of the expression of genes were analyzed with the SPSS (Version 21.0) using the Student's $t$-test analysis.

\section{Additional files}

\section{Additional file 1: Figure S1. Phylogenetic tree showing the} relationships of 43 soybean SPLs with the AtSPL9, AtSPL15, OsSPL14 and OsSPL17 proteins. (DOCX $232 \mathrm{~kb}$ )

Additional file 2: Table S1. CRISPR/Cas9-meditated targeted mutagenesis of four GmSPL9 genes in transgenic soybean plants of different generations. (DOCX $95 \mathrm{~kb}$ )

Additional file 3: Figure S2. Multiple alignment of the amino-acid sequences of $(A)$ two edited types of spl9b mutants and (B) one edited type of sp/9c mutant. (DOCX $426 \mathrm{~kb}$ )

Additional file 4: Text S1. The coding sequence (CDS) and protein sequence of GMSPL9 genes, and mutated sequences obtained by CRISPR/Cas9-mediated mutagenesis. (DOCX $381 \mathrm{~kb}$ )

Additional file 5: Figure S3. Identification of 'transgene-clean' mutant lines. (DOCX $94 \mathrm{~kb}$ )

Additional file 6: Figure S4. The spla9a/spl9b-1 double homozygous mutant plants showed slightly shorter plastochron length. (DOCX $165 \mathrm{~kb}$ )

Additional file 7: Figure S5. Effect of GmSPL9 transcription factor on expression of GmSPL9a, GmSPL9c and GmSPL9d in soybean leaves, as well as on soybean architecture. (DOCX $90 \mathrm{~kb}$ )

Additional file 8: Table S2. Primers used for PCR and $q R T-P C R$ in this study. (DOCX $84 \mathrm{~kb}$ )

\section{Abbreviations}

AM: Axillary meristem; AP2: Apetala 2; APC/C: Anaphase-promoting complex/ cyclosome; Cas9: CRISPR-associated system 9; CRISPR: Clustered regularly interspaced short palindromic repeat; CTAB: Cetyltrimethyl ammonium bromide; DEP1: DENSE AND ERECT PANICLE 1; FT: Flower Locus T; GmILPA1: Glycine max INCREASED LEAF PETIOLE ANGLE 1; IPA1: IDEAL PLANT ARCHITECTURE 1; IPI1: IPA1 INTERACTING PROTEIN 1; QTLS: Quantitative trait loci; SAM: Shoot apical meristem; SBP: SQUAMOSA PROMOTER BINDING PROTEIN; SPL: SQUAMOSA PROMOTER BINDING PROTEIN-LIKE; TB1: TEOSINTE BRANCHED 1; TF: Transcription factor; TGA1: TEOSINTE GLUME ARCHITECTURE; TSH4: TASSELSHEATH4; Ub2: UNBRANCHED2; Ub3: UNBRANCHED3; WFP: WEALTHY FARMER'S PANICLE; WT: Wild-type; WUS: WUSCHEL 


\section{Acknowledgements}

We thank Professor Yaoguang Liu from South China Agricultural University for providing the vector pYLCRISPR/Cas9P ${ }_{355}-\mathrm{B}$. We thank Dr. Kan Wang, lowa State University of Science and Technology for providing the vector pTF101.1 and Agrobacterium strain EHA101.

\section{Funding}

The research was supported by the National Genetically Modified Organisms Breeding Major Projects (2016ZX08004-005). The funding body had no role in the design of the study and collection, analysis, and interpretation of data and in writing the manuscript.

\section{Availability of data and materials}

The datasets and materials generated and analyzed during the current study are available from the corresponding author on reasonable request.

\section{Authors' contributions}

DC and L-SPT conceived and designed the experiments; $\mathrm{AB}$ and $\mathrm{HC}$ constructed the vectors; $A B, L C$ and $X Z 1$ performed the Agrobacterium-mediated transformation; $A B$, SC and QH performed the mutation analysis; WG performed the qRT-PCR analysis; DQ, ZS, ZY and SY performed the investigation of phenotypic parameter; CZ analyzed the data; XL, FK and BL generated the GmmiR156b-OX and GmSPL9bOX plants; XZ2, L-SPT, DC wrote and revised the paper. All the authors read and finalized the version before submission.

\section{Ethics approval and consent to participate}

Not applicable.

\section{Consent for publication}

Not applicable.

\section{Competing interests}

The authors declare that they have no competing interests.

\section{Publisher's Note}

Springer Nature remains neutral with regard to jurisdictional claims in published maps and institutional affiliations.

\section{Author details}

${ }^{1}$ Key Laboratory of Biology and Genetic Improvement of Oil Crops, Ministry of Agriculture, Oil Crops Research Institute, Chinese Academy of Agricultural Sciences, Wuhan 430062, China. ${ }^{2}$ The Key Laboratory of Soybean Molecular Design Breeding, Northeast Institute of Geography and Agroecology, Chinese Academy of Sciences, Harbin 150081, China. ${ }^{3}$ State Key Laboratory of Agricultural Microbiology, College of Plant Science and Technology, Huazhong Agricultural University, Wuhan 430070, China. ${ }^{4}$ Institute of Research and Development, Duy Tan University, 03 Quang Trung, Da Nang, Vietnam. ${ }^{5}$ Stress Adaptation Research Unit, RIKEN Center for Sustainable Resource Science, 1-7-22, Suehiro-cho, Tsurumi, Yokohama 230-0045, Japan.

Received: 28 December 2018 Accepted: 28 March 2019

Published online: 08 April 2019

\section{References}

1. Hartung RC, Specht JE, Williams JH. Modification of soybean plant architecture by genes for stem growth habit and maturity. Crop Sci. 1981; 21:51-6.

2. Huyghe C. Genetics and genetic modifications of plant architecture in grain legumes: a review. Agronomie. 1998:18:383-411.

3. Bernard RL. Two genes affecting stem termination in soybean. Crop Sci. 1972:12:235-9.

4. Liu BH, Watanabe S, Uchiyama T, Kong FJ, Kanazawa A, Xia ZJ, Nagamatsu A, Arai M, Yamada T, Kitamura $K$, et al. The soybean stem growth habit gene Dt1 is an ortholog of Arabidopsis TERMINAL FLOWER1. Plant Physiol. 2010;153:198-210

5. Tian ZX, Wang XB, Lee R, Li YH, Specht JE, Nelson RL, McClean PE, Qiu LJ, Ma JX. Artificial selection for determinate growth habit in soybean. P Natl Acad Sci USA. 2010;107:8563-8.

6. Ping J, Liu Y, Sun L, Zhao M, Li Y, She M, Sui Y, Lin F, Liu X, Tang Z. Dt2 is a gain-of-function MADS-domain factor gene that specifies semideterminacy in soybean. Plant Cell. 2014;26:2831-42.
7. Liu YF, Zhang DJ, Ping JQ, Li S, Chen ZX, Ma JX. Innovation of a regulatory mechanism modulating semi-determinate stem growth through artificial selection in soybean. PLoS Genet. 2016;12:e1005818.

8. Gao JS, Yang SX, Cheng W, Fu YF, Leng JT, Yuan XH, Jiang N, Ma JX, Feng $X Z$. GmILPA1, encoding an APC8-like protein, controls leaf petiole angle in soybean. Plant Physiol. 2017;174:1167-76.

9. Schwab R, Palatnik JF, Riester M, Schommer C, Schmid M, Weigel D. Specific effects of MicroRNAs on the plant transcriptome. Dev Cell. 2005;8:517-27.

10. Wang JW, Czech B, Weigel D. miR156-regulated SPL transcription factors define an endogenous flowering pathway in Arabidopsis thaliana. Cell. 2009; 138:738-49.

11. Wu G, Poethig RS. Temporal regulation of shoot development in Arabidopsis thaliana by miR156 and its target SPL3. Development. 2006:133:3539-47.

12. Xing SP, Salinas M, Hohmann S, Berndtgen R, Huijser P. miR156-targeted and nontargeted SBP-box transcription factors act in concert to secure male fertility in Arabidopsis. Plant Cell. 2010;22:3935-50.

13. Schwarz S, Grande AV, Bujdoso N, Saedler H, Huijser P. The microRNA regulated SBP-box genes SPL9 and SPL15 control shoot maturation in Arabidopsis. Plant Mol Biol. 2008:67:183-95.

14. Wang JW, Schwab R, Czech B, Mica E, Weigel D. Dual effects of miR156targeted SPL genes and CYP78A5/KLUH on plastochron length and organ size in Arabidopsis thaliana. Plant Cell. 2008;20:1231-43.

15. Jiao YQ, Wang YH, Xue DW, Wang J, Yan MX, Liu GF, Dong GJ, Zeng DL, Lu ZF, Zhu XD, et al. Regulation of OSSPL14 by OsmiR156 defines ideal plant architecture in rice. Nat Genet. 2010:42:541-4.

16. Miura K, Ikeda M, Matsubara A, Song XJ, Ito M, Asano K, Matsuoka M, Kitano $\mathrm{H}$, Ashikari M. OsSPL14 promotes panicle branching and higher grain productivity in rice. Nat Genet. 2010;42:545-9.

17. Luo L, Li WQ, Miura K, Ashikari M, Kyozuka J. Control of tiller growth of rice by OsSPL14 and strigolactones, which work in two independent pathways. Plant Cell Physiol. 2012;53:1793-801.

18. Lu ZF, Yu H, Xiong GS, Wang J, Jiao YQ, Liu GF, Jing YH, Meng XB, Hu XM, Qian $Q$, et al. Genome-wide binding analysis of the transcription activator IDEAL PLANT ARCHITECTURE1 reveals a complex network regulating rice plant ARCHITECTURE. Plant Cell. 2013;25:3743-59.

19. Wang $L$, Sun $S Y$, Jin JY, Fu DB, Yang XF, Weng XY, Xu CG, Li XH, Xiao JH, Zhang QF. Coordinated regulation of vegetative and reproductive branching in rice. P Natl Acad Sci USA. 2015;112:15504-9.

20. Wang J, Yu H, Xiong GS, Lu ZF, Jiao YQ, Meng XB, Liu GF, Chen XW, Wang YH, Li JY. Tissue-specific ubiquitination by IPA1 INTERACTING PROTEIN1 modulates IPA1 PROTEIN levels to regulate plant architecture in rice. Plant Cell. 2017;29:697-707.

21. Sun Z, Su C, Yun J, Jiang Q, Wang L, Wang Y, Cao D, Zhao F, Zhao Q, Zhang $M$, et al. Genetic improvement of the shoot architecture and yield in soybean plants via the manipulation of GmmiR156b. Plant Biotechnol J. 2018; doi.org/10.1111/pbi.12946.

22. Li JF, Norville JE, Aach J, McCormack M, Zhang DD, Bush J, Church GM, Sheen J. Multiplex and homologous recombination-mediated genome editing in Arabidopsis and Nicotiana benthamiana using guide RNA and Cas9. Nat Biotechnol. 2013;31:688-91.

23. Shan QW, Wang YP, Li J, Zhang Y, Chen KL, Liang Z, Zhang K, Liu JX, Xi JJ, Qiu JL, et al. Targeted genome modification of crop plants using a CRISPRCas system. Nat Biotechnol. 2013:31:686-8.

24. Nekrasov V, Staskawicz B, Weigel D, Jones JDG, Kamoun S. Targeted mutagenesis in the model plant Nicotiana benthamiana using Cas9 RNAguided endonuclease. Nat Biotechnol. 2013;31:691-3.

25. Zhang YW, Bai Y, Wu GH, Zou SH, Chen YF, Gao CX, Tang DZ. Simultaneous modification of three homoeologs of TaEDR1 by genome editing enhances powdery mildew resistance in wheat. Plant J. 2017:91:714-24.

26. Zhang SJ, Zhang RZ, Song GQ, Gao J, Li W, Han XD, Chen ML, Li YL, Li GY. Targeted mutagenesis using the agrobacterium tumefaciens-mediated CRISPR-Cas9 system in common wheat. BMC Plant Biol. 2018:18:302.

27. Howells RM, Craze M, Bowden S, Wallington EJ. Efficient generation of stable, heritable gene edits in wheat using CRISPR/Cas9. BMC Plant Biol. 2018;18:215.

28. Liang Z, Zhang $\mathrm{K}$, Chen $\mathrm{KL}$, Gao CX. Targeted mutagenesis in zea mays using TALENs and the CRISPR/Cas system. J Genet Genomics. 2014;41:63-8.

29. Shi JR, Gao HR, Wang HY, Lafitte HR, Archibald RL, Yang MZ, Hakimi SM, Mo H, Habben JE. ARGOS8 variants generated by CRISPR-Cas9 improve maize grain yield under field drought stress conditions. Plant Biotechnol J. 2017;15:207-16.

30. Braatz J, Harloff HJ, Mascher M, Stein N, Himmelbach A, Jung C. CRISPR-Cas9 targeted mutagenesis leads to simultaneous modification of different 
homoeologous gene copies in polyploid oilseed rape (Brassica napus). Plant Physiol. 2017;174:935-42.

31. Lawrenson T, Shorinola O, Stacey N, Li CD, Ostergaard L, Patron N, Uauy C, Harwood W. Induction of targeted, heritable mutations in barley and Brassica oleracea using RNA-guided Cas9 nuclease. BMC Genome Biol. 2015; 16:258.

32. Wang PC, Zhang J, Sun L, Ma YZ, Xu J, Liang SJ, Deng JW, Tan JF, Zhang $\mathrm{QH}$, Tu LL, et al. High efficient multisites genome editing in allotetraploid cotton (Gossypium hirsutum) using CRISPR/Cas9 system. Plant Biotechnol J. 2018;16:137-50

33. Soyk S, Muller NA, Park SJ, Schmalenbach I, Jiang K, Hayama R, Zhang L, Van Eck J, Jimenez-Gomez JM, Lippman ZB. Variation in the flowering gene SELF PRUNING 5G promotes day-neutrality and early yield in tomato. Nat Genet. 2017:49:162-8

34. Li ZS, Liu ZB, Xing AQ, Moon BP, Koellhoffer JP, Huang LX, Ward RT, Clifton E, Falco SC, Cigan AM. Cas9-guide RNA directed genome editing in soybean. Plant Physiol. 2015;169:960-70.

35. Tang F, Yang SM, Liu JG, Zhu HY. Rj4, a gene controlling nodulation specificity in soybeans, encodes a thaumatin-like protein but not the one previously reported. Plant Physiol. 2016;170:26-32.

36. Cai YP, Chen L, Liu XJ, Guo C, Sun S, Wu CX, Jiang BJ, Han TF, Hou WS, CRISPR/Cas9-mediated targeted mutagenesis of GmFT2a delays flowering time in soya bean. Plant Biotechnol J. 2018;16:176-85.

37. Cao D, Li Y, Wang JL, Nan HY, Wang YN, Lu SJ, Jiang Q, Li XM, Shi DN, Fang C, et al. GmmiR156b overexpression delays flowering time in soybean. Plant Mol Biol. 2015;89:353-63.

38. Ma XL, Zhang QY, Zhu QL, Liu W, Chen Y, Qiu R, Wang B, Yang ZF, Li HY, Lin YR, et al. A robust CRISPR/Cas9 system for convenient, high-efficiency multiplex genome editing in monocot and dicot plants. Mol Plant. 2015;8: $1274-84$.

39. Zhang H, Zhang JS, Wei PL, Zhang BT, Gou F, Feng ZY, Mao YF, Yang L, Zhang H, Xu NF, et al. The CRISPR/Cas9 system produces specific and homozygous targeted gene editing in rice in one generation. Plant Biotechnol J. 2014;12:797-807.

40. Feng ZY, Mao YF, Xu NF, Zhang BT, Wei PL, Yang DL, Wang Z, Zhang ZJ, Zheng $R$, Yang $L$, et al. Multigeneration analysis reveals the inheritance, specificity, and patterns of CRISPR/Cas-induced gene modifications in Arabidopsis. P Natl Acad Sci USA. 2014;111:4632-7.

41. Hada A, Krishnan V, Jaabir MSM, Kumari A, Jolly M, Praveen S, Sachdev A. Improved Agrobacterium tumefaciens-mediated transformation of soybean [Glycine max (L.) Merr.] following optimization of culture conditions and mechanical techniques. In Vitro Cell Dev-PI. 2018;54:672-88.

42. Liu J, Cheng XL, Liu P, Sun JQ. miR156-targeted SBP-box transcription factors interact with DWARF53 to regulate TEOSINTE BRANCHED1 and BARREN STALK1 expression in bread wheat. Plant Physiol. 2017;174:1931-48.

43. Wang H, Nussbaum-Wagler T, Li BL, Zhao Q, Vigouroux Y, Faller M, Bomblies K, Lukens L, Doebley JF. The origin of the naked grains of maize. Nature. 2005;436:714-9.

44. Chuck G, Whipple $C$, Jackson D, Hake S. The maize SBP-box transcription factor encoded by tasselsheath 4 regulates bract development and the establishment of meristem boundaries. Development. 2010;137:1243-50.

45. Chuck GS, Brown PJ, Meeley R, Hake S. Maize SBP-box transcription factors unbranched 2 and unbranched 3 affect yield traits by regulating the rate of lateral primordia initiation. P Natl Acad Sci USA. 2014;111:18775-80.

46. Kumar S, Stecher G, Tamura K. MEGA7: molecular evolutionary genetics analysis version 7.0 for biggerdatasets. Mol Biol Evol. 2016;33:1870-4.

47. Nan HY, Cao D, Zhang DY, Li Y, Lu SJ, Tang LL, et al. GmFT2a and GmFT5a redundantly and differentially regulate flowering through interaction with and upregulation of the bZIP transcription factor GmFDL19 in soybean. PLoS One. 2014;9:e97669.

Ready to submit your research? Choose BMC and benefit from:

- fast, convenient online submission

- thorough peer review by experienced researchers in your field

- rapid publication on acceptance

- support for research data, including large and complex data types

- gold Open Access which fosters wider collaboration and increased citations

- maximum visibility for your research: over $100 \mathrm{M}$ website views per year

At BMC, research is always in progress.

Learn more biomedcentral.com/submissions 